Elseviex Science Publishers B.V., Amsterdam - Printed in The Netherlands

\title{
RATE-CONTROLLED RECTAL DRUG DELIVERY IN MAN WITH A HYDROGEL PREPARATION
}

\author{
L.G.J. de Leede*, A.G. de Boer, E. Pörtzgen, \\ Dept. of Pharmaceutical Technology and Biopharmacy, University of Leiden, Leiden (The Netherlands) \\ J. Feijen, \\ Dept of Chemical Technology, Twente University of Technology, Enschede (The Netherlands) \\ and D.D. Breimer \\ Dept. of Pharmacology, University of Leiden. Leiden (The Netheriands)
}

(Received December 11, 1984; accepted in revised form October 17, 1985)

Cylindrical hydrogels of hydroxyethyl methacrylate (HEMA) and ethylene glycoldimethacrylate (EGDMA) as crosslinking agent were prepared by radical polymerization at $70^{\circ} \mathrm{C}$. After washing they were soaked in an aqueous drug solution of antipyrine or theophylline. The in vitro drug release experiments were performed in $100 \mathrm{ml}$ isotonic glucose at $37^{\circ} \mathrm{C}$. Rectal administration of a hydrogel preparation containing antipyrine was performed in two subjects for $72 \mathrm{~h}$. With a theophylline-containing hydrogel preparation rectal drug administration was performed in six volunteers for $24 \mathrm{~h}$. Plasma and saliva samples were taken regularly and the in vivo drug release was determined by means of a deconvolution procedure. In vitro $1.12 \mathrm{~g}$ antipyrine had been released according to a matrix-type profile for $72 \mathrm{~h}$, whereas it was calculated that this was 1.13 and $1.09 \mathrm{~g}$ in vivo in the two subjects. The release profile in vivo was very similar to that in vitro. The theophylline hydrogel preparation released in vitro a total of $288 \pm 6 \mathrm{mg}$ of drug in $24 \mathrm{~h}$ and in vivo this amount was calculated to be $288 \pm 11 \mathrm{mg}$ (mean \pm s.d.). Near-constant plasma theophylline concentrations were obtained after administering the hydrogel preparation. In all six subjects the cumulative drug profile was in almost perfect agreement with that observed in vitro. Hydrogels offer interesting perspectives as rate-controlled rectal drug delivery systems because of the predictable release profile in vivo on the basis of observations in a simple in vitro model.

\section{INTRODUCTION}

Hydrogels, defined as hydrophilic polymers which are able to contain a considerable amount of water without dissolving, form an important class of polymeric materials which can be used as drug delivery systems. Since

*To whom correspondence should be addressed; present address: Pharm. R\&D, Organon Int., P.O. Box 20,5340 BH Oss, The Netherlands. the introduction of a synthetic hydrogel, poly(hydroxyethyl methacrylate) (poly-HEMA) in 1960 [1], extensive research has been performed in the area of biomedical use of hydrogels $[2,3]$. Such investigations aim at the preparation of well-controlled rather than sustained-release delivery systems containing bioactive agents. For a rate-controlled drug delivery system a consistent correlation between the release characteristics in vivo and those in vitro is considered to be the most im. 
portant feature. Investigations with controlledrelease hydrogel preparations with a relatively short period of drug release are scarce. Very few studies have been published in which nondisintegrating hydrogel matrices were designed to be administered by the oral or rectal route [4-7]. A hydrogel-procainamide preparation has been given orally to rabbits, which resulted in a prolonged release profile compared to a conventional capsule. Morphineloaded poly(ethyleneglycol)-base hydrogels administered rectally to four patients exhibited near constant and prolonged drug delivery, but in vivo release was found to be slower than in vitro. The rectal route is very suitable for drug administration [8], and in previous studies a rate-controlled osmotic drug delivery system was successfully applied in healthy subjects [ $9-12]$.

In contrast to data in vivo the in vitro release characteristics of drugs and solutes from hydrogels of HEMA and ethylene glycol dimethacrylate (EGDMA) as crosslinking agent have been described extensively [1315]. In the present study in vivo investigations with hydrogels of the HEMA and EGDMA type containing antipyrine or theophylline as active substance are described. Their plasma and saliva level profiles were studied after rectal administration in healthy volunteers. Hydrogel drug preparations can be made by mixing the drug with the monomer mixture followed by polymerization, or by impregnating the hydrogel with drug solutions. With the latter soaking technique possible residual monomers or initiator can be removed from the system before drug loading $[6,13,16]$ and therefore this method is to be preferred for the preparation of drug delivery systems.

Hydrogels were made with antipyrine as a model compound since it is highly watersoluble and well absorbed rectally, while plasma sampling can be replaced by saliva sampling [9]. Theophylline was used (as the water-soluble choline salt) since it is frequently applied rectally in actual practice and has a relatively low therapeutic index $[8,10]$. With conventional oral and rectal dosage forms and even with some of the sustained- release or retard formulations an optimal and in vivo predictable plasma concentration-time profile is not achieved. A relatively constant rate of drug input would be most desirable in theophylline prophylactic therapy.

\section{MATERIALS AND METHODS}

\section{Materials}

Ultrapure HEMA (Hydromed. Sci., New Brunswick, NJ, U.S.A.), EGDMA (Fluka AG, Buchs, Switzerland) and 2,2'-azobisisobutyronitrile (AIBN) (Polysciences, Warrington, PA, U.S.A.) were used. HEMA contained $0.04 \%$ methacrylic acid, 0.15\% EGDMA and 0.02\% diethylene glycol methacrylate, and was used as received. EGDMA was purified by extraction with $1 M \mathrm{NaOH}$ several times, washing with water and drying afterwards. Antipyrine and choline theophyllinate, pharmacopoeial quality (Brocacef, Maarssen, The Netherlands), were used as received.

\section{Preparation of hydrogels}

Hydrogel preparation no. I consisted of a monomer mixture of HEMA/EGDMA 100/1 $(\mathrm{w} / \mathrm{w})$ with 20 weight percent of water and preparation no. II of a HEMA/EGDMA 40/1 mixture with 40 weight percent of water. Both solutions were polymerized after degassing, in the presence of $7.84 \mathrm{mmol}$ AIBN per liter monomer mixture in a cylindrical glass mold of $11 \mathrm{~mm}$ i.d. at $70^{\circ} \mathrm{C}$ for $15 \mathrm{~h}$. After polymerization the hydrogels were rinsed with water at $37^{\circ} \mathrm{C}$ for 4 weeks and dried afterwards at $100^{\circ} \mathrm{C}$ for 4 days. Hydrogel preparation no. I was soaked in an aqueous solution of antipyrine $(150 \mathrm{mg} / \mathrm{ml})$ and no. II in an aqueous choline theophyllinate solution (180 $\mathrm{mg} / \mathrm{ml})$ at room temperature until equilibrium was reached. This was determined by in vitro release experiments. Equilibrium was assumed to have been reached when no further increase in the total amount of released drug was observed. Before the drug release experiments cylinders of the desired length $(34 \mathrm{~mm})$ were cut. 


\section{In vitro release experiments}

The in vitro release experiments were performed in a glass flask equipped with a magnetic stirrer in $100 \mathrm{ml}$ isotonic solution $(5.5 \%$ glucose) at $37.0 \pm 0.2^{\circ} \mathrm{C}$. Since the model drugs are highly water-soluble, sink conditions were always maintained. At regular time intervals samples were taken and replaced by the same volume of glucose solution; drug concentrations were determined spectrophotometrically (Perkin Elmer DB 124).

\section{Subjects and drug administration}

Eight healthy male volunteers, age 22-34, weight $60-78 \mathrm{~kg}$, participated after medical examination. All had given informed consent. Two of them participated in the antipyrine study and six in the study with theophylline. The latter study was approved by the Committee for Medical Ethics of the University Hospital and the faculty of Medicine, University of Leiden.

In the antipyrine study one hydrogel drug preparation was kept in the rectum for $72 \mathrm{~h}$. Drug administration started at 9 a.m.; saliva samples were collected at regular time intervals during $96 \mathrm{~h}$. When the subjects had to empty their bowels during the $72 \mathrm{~h}$ of drug administration, the hydrogel was recovered, cleaned and inserted again.

Rectal drug administration of the theophylline-containing hydrogel preparation took place during $24 \mathrm{~h}$. Drug administration started at $9 \mathrm{a} . \mathrm{m}$. and after $12 \mathrm{~h}$ the system was removed and replaced by a second one, which was kept in the rectum for another $12 \mathrm{~h}$. Blood samples were taken just before rectal application and after $0.25,0.5,0.75,1,1.5$, $2,3,4,6,8,10,12,15,19,22,24,28,31,34$ and $37 \mathrm{~h}$ from a cannula in a forearm vein. Plasma samples were obtained by centrifuging the heparinized blood samples immediately after blood sampling. Saliva samples were collected at regular time intervals in the period from 14 to $40 \mathrm{~h}$ when blood sampling was less frequent. Plasma and saliva samples were stored at $-20^{\circ} \mathrm{C}$ until assayed. The hydrogels were administered, if possible, after defecation. When the subjects had a bowel movement during the $24 \mathrm{~h}$ of drug administration the same procedure was followed as described above. The intake of caffeine was prohibited from $24 \mathrm{~h}$ before drug administration onwards until the last sample had been collected.

\section{Assay of antipyrine and theophylline in saliva and plasma}

The antipyrine and theophylline concentrations were determined according to previously described methods $[9,10]$, except that with the assay of theophylline, antipyrine was used as an internal standard and the chromatographic conditions were modified: a radialpak $\mathrm{C} 8$ reverse phase column with an eluent of $0.03 M$ acetate buffer $(\mathrm{pH} 4.0)$ with $4 \%$ tetrahydrofuran. Linear calibration curves were obtained from 0.2 to $10 \mu \mathrm{g}$ theophylline/ml saliva or plasma.

\section{Calculations and pharmacokinetic analysis}

The total dose administered rectally was calculated from the difference between the amount in the hydrogel before application and the amount remaining after application. The latter amount was determined by in vitro release afterwards until no further increase in the drug concentration could be found.

The total area under the plasma or saliva concentration-time curves (AUC) was calculated according to a method described by Chiou [17]. The clearance values were determined by dividing the total dose administered by AUC. The elmination rate constant $\left(k_{\mathrm{el}}\right)$ was obtained by regression analysis of the logarithm of plasma or saliva concentration against time after withdrawal of the hydrogel. Elimination half-lives $\left(t_{1 / 2} \mathrm{el}\right)$ were calculated as $t_{1 / 2 \mathrm{el}}=0.693 / k_{\mathrm{el}}$.

Each time a sample was taken the cumulative amount released in vivo was determined by means of a deconvolution procedure 
(point area method) which calculated the actual input by relating the input to a unit impulse response function [18]. For each individual this function was calculated from the $k_{\text {el }}$ and the clearance as determined at the end of the rectal experiment.

\section{RESULTS}

The in vitro release of antipyrine and theophylline from hydrogel preparations I and II were measured under stirred and non-stirred conditions. There were no significant differences between continuous stirring and stirring for $10 \mathrm{~s}$ once every hour just before sample taking. The very reproducible drug release from preparation I in isotonic glucose was in agreement with a matrix-type profile and after $72 \mathrm{~h} 82 \pm 2 \%(1.12 \mathrm{~g} ; n=3)$ of the total amount of antipyrine was present in the dis-
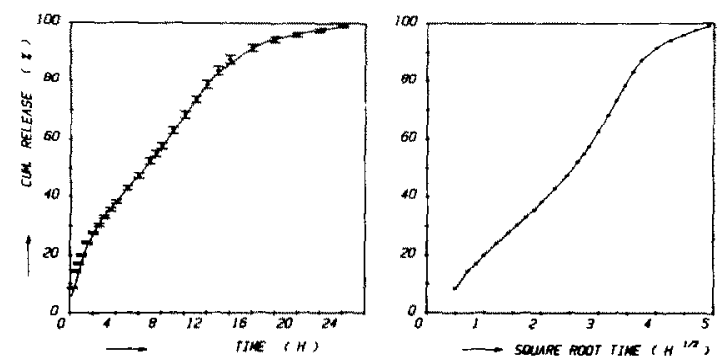

Fig. 1. Mean cumulative in vitro release of theophylline from a hydrogel preparation versus time (with s.d.) and versus the square root of lime $(n=5)$.

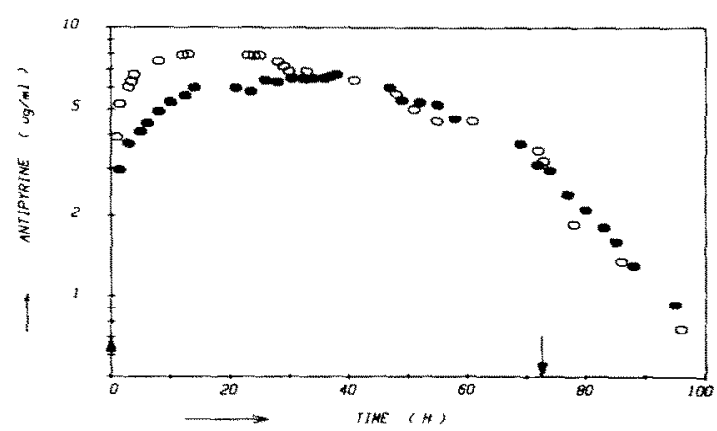

Fig. 2. Saliva concentration time profile of antipyrine after rectal administration of an antipyrine hydrogel preparation during $72 \mathrm{~h}$ in subjects $\mathrm{I}(0)$ and II (•). solution medium. The in vitro drug release from preparation II containing theophylline is shown in Fig. 1 . In $12 \mathrm{~h}$ the hydrogel released $144 \pm 3 \mathrm{mg}$ (calculated as theophylline) which was $80 \%$ of the total amount in the dosage form.

Figure 2 shows the saliva concentrationtime curves of antipyrine after rectal administration of the hydrogel preparation to the two

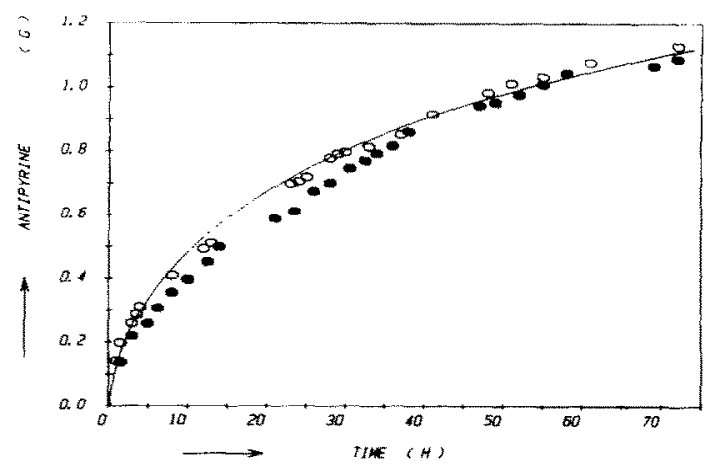

Fig. 3. Cumulative amount of antipyrine released from a hydrogel preparation in vitro (solid line) and in vivo after rectal application during $72 \mathrm{~h}$ in subject I (०) and II (•).
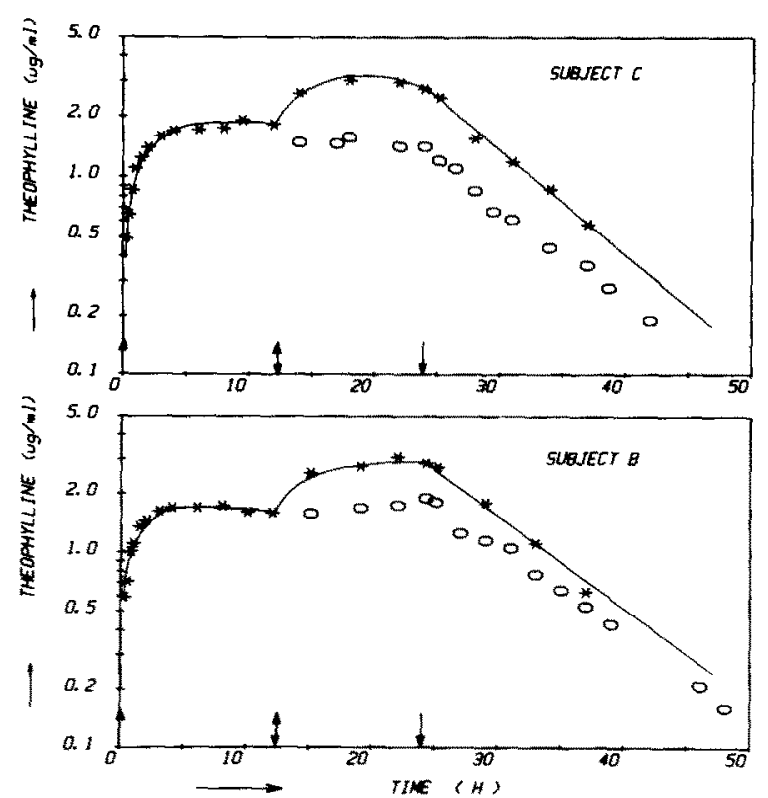

Fig. 4. Plasma (*) and saliva ( $)$ theophylline concentration time profiles after rectal administration of a hydrogel preparation to subjects $B$ and $C$. Time of insertion ( $\uparrow)$, renewal $(\downarrow)$ and withdrawal $(\downarrow)$. 
subjects. The administered dose was 1.13 and $1.09 \mathrm{~g}$ antipyrine for subjects I and II, respectively; the AUC, clearance and $k_{\text {el }}$ were 0.52 and $0.44 \mathrm{~g} \mathrm{~h} / \mathrm{l}, 2.2$ and $2.5 \mathrm{l} / \mathrm{h}$, and 0.065 and $0.047 \mathrm{~h}^{-1}$ respectively. Figure 3 shows the cumulative amount of antipyrine released in vivo, as calculated with the deconvolution method, together with the release profile in vitro (solid line). A very good agreement of the antipyrine release in vivo with that in vitro is obtained. With this drug delivery system the amount and rate of release of antipyrine after rectal application in man seems to be quite predictable on the basis of in vitro experiments.

The theophylline concentration-time curves in plasma and saliva after rectal administration of the hydrogels to subject $B$ and

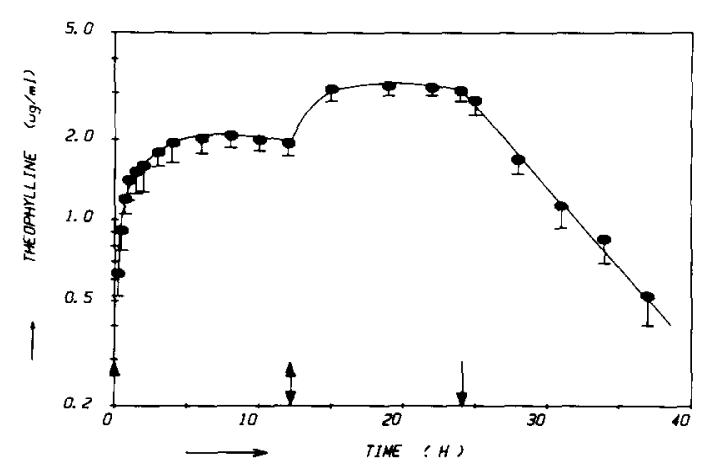

Fig. 5. Mean plasma concentration time profile after rectal administration of a theophylline hydrogel preparation (with s.d.; $n=6$ ). Time of insertion ( $\uparrow$ ), renewal $(\downarrow)$ and withdrawal $(\downarrow)$.
$\mathrm{C}$ are given in Fig. 4. A rapid increase in plasma concentration was found for the first 4 to $5 \mathrm{~h}$ and from then onwards the concentration remained almost constant until the hydrogel preparation was replaced by a second one. Again the plasma concentrations rose relatively rapidly for 4 to $5 \mathrm{~h}$ and then remained nearly constant until the dosage form was removed. During the period of almost constant theophylline concentrations and during the elimination phase the saliva plasma concentration ratio was reasonably constant. The plasma concentration-time curves were quite similar in all six subjects; in Fig. 5 the mean curve with standard deviations is shown. In Table 1 the administered dose, AUC, $k_{\mathrm{el}}$ and $t_{1 / 2 \mathrm{el}}$ are given together with the calculated clearance values. The mean elimination halflife was $5.1 \mathrm{~h}$ and the mean clearance value $3.7 \mathrm{l} / \mathrm{h}$. The mean dose administered rectally with the hydrogel preparations during $24 \mathrm{~h}$ was $288 \pm 11 \mathrm{mg}$, which corresponds perfectly with the in vitro results. Figure 6 shows the cumulative in vivo release of theophylline, calculated according to the deconvolution method, in all six subjects together with the mean in vitro release profile (solid line). In all cases the profile in vivo showed an almost perfect agreement with the situation found in vitro in isotonic glucose.

The hydrogels with theophylline or antipyrine were well tolerated by all subjects and no complaints of inconvenience or irritation were reported.

\section{TABLE 1}

Calculated doses and pharmacokinetic data following rectal administration of a theophylline-containing hydrogel preparation to six healthy volunteers

\begin{tabular}{|c|c|c|c|c|c|c|c|}
\hline & $\begin{array}{l}\text { dose } 0-12 \mathrm{~h} \\
(\mathrm{mg})\end{array}$ & $\begin{array}{l}\text { dose } 12-24 \mathrm{~h} \\
(\mathrm{mg})\end{array}$ & $\begin{array}{l}\text { total dose } \\
\text { (mg) }\end{array}$ & $\begin{array}{l}k_{\mathrm{el}} \\
\left(\mathrm{h}^{-1}\right)\end{array}$ & $\begin{array}{l}\text { AUC } \\
(\mu \mathrm{g} \mathrm{h} / \mathrm{ml})\end{array}$ & $\begin{array}{l}t_{1 / 2 \mathrm{el}} \\
(\mathrm{h})\end{array}$ & $\begin{array}{l}\text { clearance } \\
(\mathrm{l} / \mathrm{h})\end{array}$ \\
\hline $\mathbf{A}$ & 142 & 152 & 294 & 0.144 & 94.3 & 4.8 & 3.1 \\
\hline $\mathbf{B}$ & 140 & 132 & 272 & 0.107 & 77.2 & 6.5 & 3.5 \\
\hline $\mathrm{C}$ & 136 & 150 & 286 & 0.121 & 75.7 & 5.7 & 3.8 \\
\hline $\mathrm{D}$ & 149 & 152 & 301 & 0.173 & 89.3 & 4.0 & 3.4 \\
\hline $\mathrm{E}$ & 145 & 150 & 295 & 0.133 & 67.5 & 5.2 & 4.4 \\
\hline$F$ & 133 & 146 & 279 & 0.150 & 70.8 & 4.6 & 3.9 \\
\hline mean \pm s.d. & $141 \pm 6$ & $147 \pm 8$ & $288 \pm 11$ & $0.138 \pm 0.021$ & $79.1 \pm 10.5$ & $5.1 \pm 0.8$ & $3.7 \pm 0.4$ \\
\hline
\end{tabular}



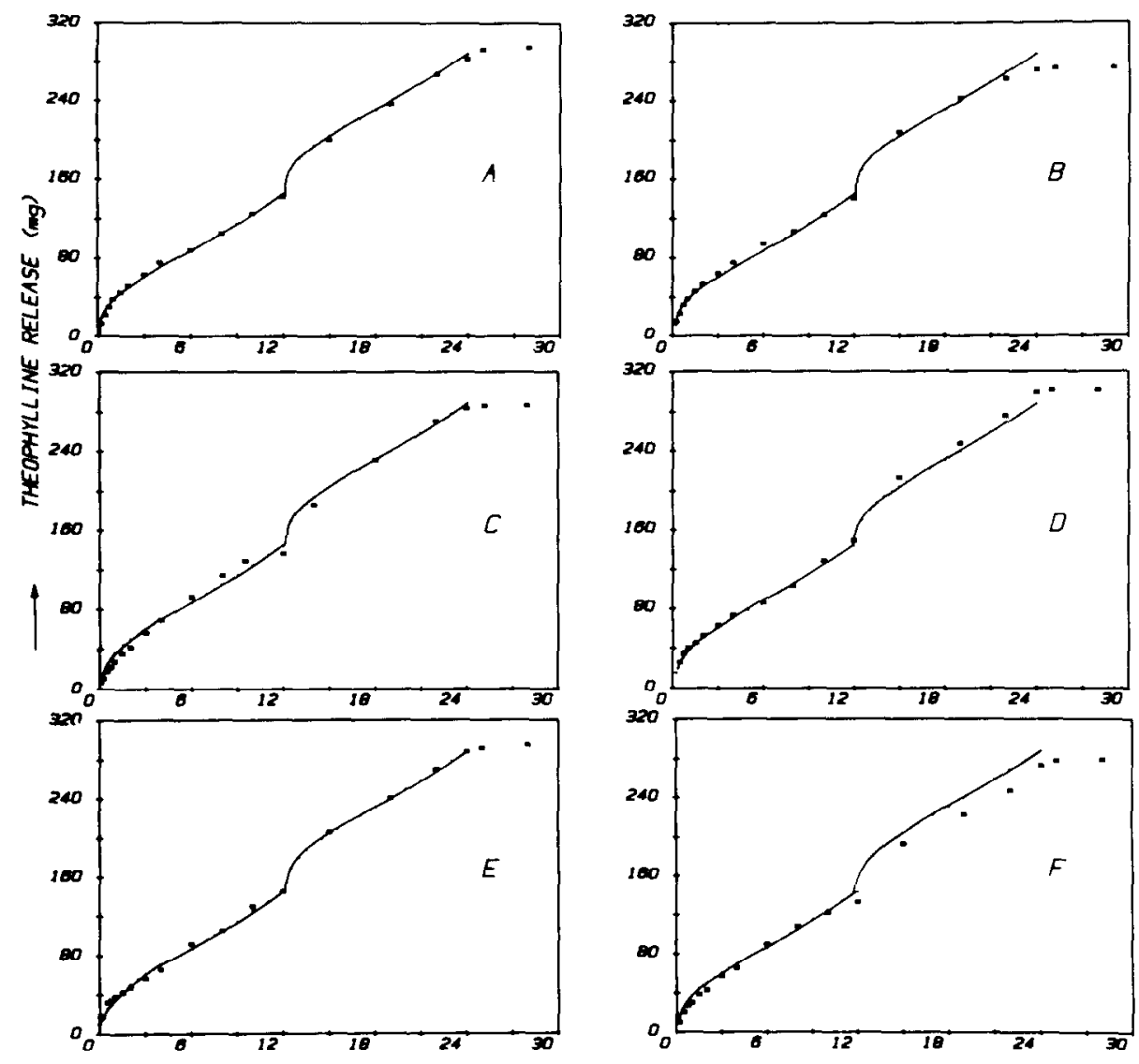

$\longrightarrow$ TIME (H)

Fig. 6. Cumulative amount of theophylline released from two hydrogels subsequently, each during $12 \mathrm{~h}$, in vitro (solid line) and in vivo (•) after rectal application, in all individuals.

\section{DISCUSSION}

The results of the present study clearly indicate that it is quite possible to obtain a reproducible and predictable in vivo drug release profile with a hydrogel drug delivery system. Since the release characteristics can be varied quite easily by for instance the geometry, the diameter, the crosslinking agent, the amount of crosslinker and the water content $[6,14,19,20]$, hydrogels offer interesting perspectives for rate-controlled rectal delivery of several drugs.

With the hydrogel preparation containing theophylline, with a relatively high drug load and crosslinker percentage, an almost constant release was obtained for some time, leading to quite constant plasma concentration levels. However, the drug release profile from the hydrogels is of a matrix type, which means that the release rate decreases continuously. In principle there are possibilities to get a zero-order rate of release from hydrogeis when drugs with short elimination half-lives other than our model drugs, are used. Lee et al. [21] introduced rate-controlling barriers by soaking the hydrogel outer layer with crosslinker followed by irradiation. A zeroorder release of progesterone in vitro was demonstrated. Also a change in geometry to 
cylinder-type sections or to hemispheric matrices coated with an impermeable layer except for an exposed cavity in the centre face, can be used to achieve nearly zero-order release kinetics in vitro $[19,22]$. Using swelling systems a flattening of the rclease rate versus time profile can be obtained [7].

In the present investigation the hydrogels were loaded with drugs by means of a soaking technique, which is rather time-consuming. This procedure was chosen in order to be able to wash the hydrogels after polymerization to ensure that no monomers or initiator were present in the final preparations $[6,13,16]$. When the drug is dissolved in the monomer mixture before polymerization, the hydrogels cannot be washed without loss of drug. Whether the impregnation or soaking technique could be further improved or accelerated remains the subject of further studies.

The antipyrine and theophylline elimination half-lives and clearance values obtained after application of the rectal drug delivery systems were comparable to previously obtained data $[9,10]$ and to data described in the literature [23-25]. The deconvolution method used to describe the in vivo drug release profile requires an impulse response function which was derived from the experimental clearance values and elimination rate constants. In this procedure it is assumed that clearance and elimination rate constant are constant throughout the experiment, absorption is complete and that the dose entering the systemic circulation equals the total cumulative amount released in vivo. These assumptions are not unreasonable, because both antipyrine and theophylline (as choline salt), when administered in solution and at a relatively slow rate, are well absorbed rectally $[9,10]$. An i.v. experiment performed at the same time as the rectal one, for instance by using theophylline and labelled theophylline, would theoretically lead to a better impulse response function. However, since large intraindividual differences in clearance may exist, with changes of up to $40 \%$ within a few weeks $[10,25]$, an i.v. experiment before or after the rectal experiment may not be very meaningful.

\section{CONCLUSION}

The release characteristics of both antipyrine and theophylline from a hydrogel preparation of HEMA crosslinked with EGDMA in vivo, when applied rectally in healthy volunteers, were almost identical to those observed in vitro. It is evident that hydrogel systems, applicable to a large number of different drugs, have great potential as flexible rectal drug delivery systems. Large scale production and in vivo studies with different drugs should reveal the possibilities of replacing conventional rectal dosage forms by synthetic hydrogel drug preparations.

\section{REFERENCES}

1 O. Wichterle and D. Lim, Hydrophilic gels for biomedical use, Nature, 185 (1960) 117-118.

2 J. Andrade, Hydrogels for Medical and Related Applications, American Chemical Society, Symposium Series No. 31, ACS, Washington, DC, 1976.

3 R.S. Langer and N.A. Peppas, Present and future applications of biomaterials in controlled drug delivery systems, Biomaterials, 2 (1981) 201 214.

4 W.R. Good, in: R.J. Kostelnik (Ed.), Polymeric Delivery Systems, Gordon \& Breach, New York, NY, 1976, pp. 139-156.

5 W.R. Good and K.F. Mueller, in: R. Baker (Ed.), Controlled Release of Bioactive Materials, Academic Press, New York, NY, 1980, pp. 155183.

6 P. Gijselinck, E. Schacht, R. Van Severen and P. Braeckman, Preparation and characterization of therapeutic hydrogels as oral dosage forms, Acta Pharm. Technol., 29 (1983) 9-12.

7 N.B. Graham and M.E. McNeill, Hydrogels for controlled drug delivery, Biomaterials, 5 (1984) $27-36$.

8 A.G. de Boer, F. Moolenaar, L.G.J. de Leede and D.D. Breimer, Rectal drug administration: Clinical pharmacokinetic considerations, Clin. Pharmacokin., 7 (1982) 285--311.

9 L.G.J. de Leede, A.G. de Boer and D.D. Breimer, Rectal infusion of the model drug antipyrine with an osmotic delivery system, Biopharm. Drug Disp., 2 (1981) 131-136.

10 L.G.J. de Leede, A.G. de Boer, S.L. van Velzen and D.D. Breimer, Zero-order rectal delivery of theophylline in man with an osmotic system, J. Pharmacokin. Biopharm., 10 (1982) 525-537.

11 L.G.J. de Leede, A.G. de Boer, C.D. Feyen and 
D.D. Breimer, Site-specific rectal drug administration in man with an osmotic system, Pharm. Res., 1 (3) (1984) 129-134.

12 L.G.J. de Leede, C.C. Hug, Jr., S. de Lange, A.G. de Boer and D.D. Breimer, Rectal and intravenous infusion of propranolol to steady state: Kinetics and $\beta$-receptor blockade, Clin. Pharmacol. Ther., 35 (1984) 148-155.

13 J.M. Wood, D. Attwood and J.H. Collett, The swelling properties of poly (2-hydroxyethyl methacrylate) hydrogels polymerized by gammairradiation and chemical initiation, Int. J. Pharm., 7 (1981) 189-196.

14 G.M. Zentner, J.R. Cardinal, J. Feijen and S.-Z. Song, Progestin permeation through polymer membranes. IV, J. Pharm. Sci., 68 (1979) 970975 .

15 C. Migliaresi, L. Nicodemo, L. Nicolais and P. Passerini, Water sorption and desorption in 2hydroxyethylmethacrylate/methylmethacrylate copolymers, Polymer, 25 (1984) 686-689.

16 D.J. Lamb, B. Ellis and D. Priestley, Loss into water of residual monomer from autopolymerizing dental acrylic resin, Biomaterials, 3 (1982) 155-159.

17 W.L., Chiou, Critical evaluation of the potential error in pharmacokinetic studies of using the linear trapezoidal rule method for the calculation of the area under the plasma level time curve, J. Pharmacokin. Biopharm., 6 (1978) 539-547.

18 D.P. Vaughan and M. Dennis, Mathematical basis of point-area deconvolution method for determining in vivo input functions, J. Pharm. Sci., 67 (1978) 663-665.

19 D. Brooke and R.J. Washkuhn, Zero-order drug delivery systems: Theory and preliminary testing, J. Pharm. Sci., 66 (1977) 159-162.

20 J.M. Wood, D. Attwood and J.H. Collett, The influence of gel formulation on the diffusion of salicylic acid in poly-HEMA hydrogels, J. Pharm. Pharmacol., 34 (1982) 1-4.

21 E.S. Lee, S.W. Kim, S.H. Kim, J.R. Cardinal and H. Jacobs, Drug release from hydrogel devices with rate-controlling barriers, J. Membrane Sci., 7 (1980) 293-303.

22 D.S.T. Hsieh, W.D. Rhine and R. Langer, Zeroorder controlled-release polymer matrices for micro- and macromolecules, J. Pharm. Sci., 72 (1983) 17-22.

23 R.I. Ogilvie, Clinical pharmacokinetics of theophylline, Clin. Pharmacokin., 3 (1978) 267293.

24 L. Hendeles, M. Weinberger and L. Bighley, Absolute bioavailability of oral theophylline, Amer. J. Hosp. Pharm., 34 (1977) 525-527.

25 R.A. Upton, J.F., Thiercelin, T.W. Guentert, S.M. Wallace, J.R. Powell, L. Sansom and S. Riegelman, Intraindividual variability in theophylline pharmacokinetics: Statistical verification in 39 of 60 healthy young adults, J. Pharmacokin. Biopharm., 10 (1982) 123-134. 